\title{
CÁNCER DE PULMÓN, UNA REVISIÓN SOBRE EL CONOCIMIENTO ACTUAL, MÉTODOS DIAGNÓSTICOS Y PERSPECTIVAS TERAPÉUTICAS
}

\author{
Edgar Amorín Kajatt1 ${ }^{1,2, a}$
}

\author{
RESUMEN
}

\begin{abstract}
A nivel mundial, el cáncer de pulmón es el cáncer más frecuente en ambos sexos, seguido del cáncer de mama, colon y recto, estómago e hígado. Afecta con más frecuencia a las personas entre los 50 a 60 años, siendo el tabaquismo el factor de riesgo más asociado al desarrollo de este tipo de cáncer. Es más frecuente entre las personas de sexo masculino, sin embargo la prevalencia en el sexo femenino se ha incrementado en los últimos diez años. En el diagnóstico por imágenes destacan la tomografía por emisión de positrones con fusión a la tomografía computarizada y la econosonografía broncoscópica y transesofágica. Entre las técnicas de diagnóstico precoz sobresale la tomografía helicoidal de dosis bajas, sin embargo su uso aún no ha probado ser útil como herramienta de tamizaje. Los métodos convencionales usados para la confirmación del diagnóstico de cáncer de pulmón son la broncoscopía y la biopsia percutánea por aspiración. El tipo histológico más frecuente es el adenocarcinoma, siendo los estadios clínicos III y IV los encontrados más comúnmente. En estadios clínicos tempranos el tratamiento quirúrgico ha probado ser efectivo y tener una buena sobrevida a 5 años. En estadios avanzados, la quimioterapia y radioterapia son las modalidades más útiles para el control de la enfermedad y paliar signos y síntomas del cáncer. Los avances en análisis genómico pueden proporcionar un mejor entendimiento de la genética del cáncer y con ello nuevos blancos terapéuticos a futuro.
\end{abstract}

Palabras clave: Neoplasias pulmonares; Epidemiología; Diagnóstico; Terapia (fuente: DeCS BIREME).

\section{LUNG CANCER: A REVIEW OF CURRENT KNOWLEDGE, DIAGNOSTIC METHODS AND THERAPEUTIC PERSPECTIVES}

\section{ABSTRACT}

\begin{abstract}
All around the world, lung cancer is the most common cancer among men and women, followed by breast, colon and rectum, stomach, and liver cancer. It affects most frequently people in their 50 s and 60 s, and tobacco consumption is the risk factor most strongly associated to the development of this type of cancer. It is most common among men; however, the prevalence among women has increased over the last ten years. Imaging tests for diagnosis include positron emission tomography (PET) with computed-tomography fusion, and transesophageal and bronchoscopic ultrasound, as the most relevant. Among the early detection techniques, the low-dose helical tomography stands out. However, it has not been able to prove to be useful as a screening tool. Conventional methods for diagnostic confirmation of lung cancer are bronchoscopy and percutaneous needle aspiration biopsy. The most common histologic type is the adenocarcinoma, being clinical stages III and IV being the most frequently found. At early clinical stages, surgical treatment has proven to be effective, and there is a good 5-year survival rate. In late stages, chemotherapy and radiotherapy are the most useful treatment methods to control the disease and palliate cancer signs and symptoms. Research in genomic analysis may provide a better understanding of cancer genetics, and therefore, new therapeutic strategies in the future.
\end{abstract}

Key words: Lung neoplasms; Epidemiology; Diagnosis; Therapy (source: MeSH NLM).

\section{INTRODUCCIÓN}

El cáncer de pulmón es una enfermedad mortal cuando se diagnostica en estadios clínicos avanzados. Infortunadamente, debido a los síntomas inespecíficos de esta enfermedad en sus estadios tempranos, para cuando los pacientes acuden a la consulta generalmente el cáncer de pulmón se encuentra en estadio IIIB o IV, que implica una pobre sobrevida a cinco años; es por ello que las tendencias actuales y a futuro, respecto al

\footnotetext{
Instituto Nacional de Enfermedades Neoplásicas. Lima, Perú.

2 Clínica Ricardo Palma. Lima, Perú.

a Cirujano oncólogo, cirujano de tórax y cardiovascular, doctor en Medicina.

Recibido: 13-01-13 Aprobado: 20-02-13
}

Citar como: Amorín Kajatt E. Cáncer de pulmón, una revisión sobre el conocimiento actual, métodos diagnósticos y perspectivas terapéuticas. Rev Peru Med Exp Salud Publica. 2013;30(1):85-92. 
cáncer de pulmón están dirigidas a realizar políticas de prevención en la población; a promover estilos de vida saludables; a fomentar el cese del consumo de tabaco, particularmente en jóvenes y adultos jovenes; a difundir la práctica habitual de ejercicios y llevar una dieta saludable; a evitar el contacto con humo de segunda mano; a evitar exponerse a tóxicos ambientales, y a promover los cuidados y la protección ocupacional frente a cancerígenos ${ }^{(1)}$

Por otro lado, los nuevos conocimientos moleculares y genéticos, el desarrollo tecnológico, las nuevas evidencias epidemiológicas, los nuevos modelos de diagnóstico y las nuevas armas terapéuticas han permitido un mejor control de la enfermedad, con perspectivas de encontrar una solución favorable en un plazo mediato. En etapas muy tempranas la cirugía es curativa en altos porcentajes; con el advenimiento de la cirugía mínimamente invasiva; la cirugía por videotoracoscopía y la robótica se ha logrado minimizar el dolor, disminuir el número de días de hospitalización y mejorar la sobrevida. Hoy en día, además, se cuenta con nuevas modalidades de tratamiento no quirúrgico con preservación de órgano, como son: la radiación dirigida y el tratamiento biológico (empleando nuevos blancos terapéuticos); la reparación del DNA dañado y su restitución (con el uso de los Microarrays) es el futuro promisor en el manejo futuro del cáncer de pulmón. La nanotecnología es aún promisoria en el campo de la oncología, sin embargo, en Europa ya se vienen trabajando con nanopartículas para detectar y tratar el cáncer de pulmón a nivel molecular.

Por todo ello, es necesario conocer la epidemiología del cáncer de pulmón, sin dejar de lado los aspectos genéticos y moleculares que forman parte de la génesis, desarrollo, pronóstico y sobrevida de esta enfermedad. Se debe conocer cuáles son las nuevas técnicas de diagnóstico, su aplicación, ventajas y beneficios, enfatizando en aquellas que ayuden a un diagnóstico precoz. No se deben dejar de mencionar las alternativas terapéuticas y los avances que en este campo se han logrado.

\section{EPIDEMIOLOGÍA}

En el mundo, el cáncer más frecuente en ambos sexos es el cáncer de pulmón seguido de mama, colon y recto, estómago, e hígado ${ }^{2)}$; en el sexo masculino el cáncer de pulmón ocupa el primer lugar, luego le sigue la próstata, colon y recto, estómago, e hígado; en el sexo femenino encabeza el cáncer de mama, seguido de colon y recto, cuello uterino, pulmón, y estómago. En el 2004, la incidencia de cáncer de pulmón fue de 1092056 casos nuevos en varones en el mundo, en mujeres este valor fue de 427 586, que representa algo menos de la mitad de la incidencia en varones; para este mismo año la mortalidad registrada fue de 948993 varones y 427586 mujeres, 22,5 y $12,8 \%$ del total de casos en varones y mujeres respectivamente ${ }^{(3)}$.

En Latinoamérica, el cáncer es la tercera causa de muerte y la incidencia de los tipos de cánceres es variable en cada país, tanto en el total como por sexo. El cáncer de pulmón en el Perú se encuentra entre los cinco tipos de cáncer más frecuentes. La relación de cáncer entre el varón y la mujer es casi equivalente y de continuar la tendencia de crecimiento, las mujeres podrían superar a los hombres en los siguientes años ${ }^{(4)}$.

El carcinógeno más importante para el cáncer del pulmón es el cigarrillo y hay vinculación directa de hasta el $90 \%$ en los hombres y $85 \%$ en las mujeres; el riesgo relativo para el cáncer de pulmón es de 17,2 para los hombres y 11,6 para las mujeres ${ }^{(5)}$. El humo del cigarrillo contiene más de sesenta reconocidos cancerígenos, entre los más conocidos estan las nitrosaminas, los benzopirenos y los radioisótopos del radón, todos ellos con la capacidad de alterar el ADN y, por consiguiente, contribuir en la carcinogénesis ${ }^{(5)}$. Es por ello que hoy en día es aceptado que la población en riesgo para cáncer de pulmon esta constituida por aquellas personas de más de 50 años que hayan fumado al menos 20 cigarrillos por día durante por lo menos 10 años, mas aun si tienen carga familiar de cáncer de pulmón ${ }^{(6)}$.

Por otro lado, se ha documentado a través de estudios epidemiológicos, la vinculación existente entre el humo de leña doméstico y el cáncer de pulmón, debido a las partículas nocivas como los fenoles, la acroleína, los cresoles, el acetaldehído, los compuestos orgánicos como el benceno, el formaldehido, el butadieno, e hidrocarburos aromáticos policíclicos (7). Los efectos cancerígenos luego de la exposición del humo de la madera o excrementos de animales (bosta) tienen efectos similares sobre el p53 y expresión de la proteína MDM2 ${ }^{(7)}$. El radón es otro cancerígeno conocido, que se produce cuando el uranio se descompone de manera natural en el suelo, piedras y agua; es incoloro, inodoro e insípido y, además, radioactivo. La Agencia de Protección Ambiental afirma que uno de cada 15 hogares en los EE. UU. tiene los niveles de radón por encima de los niveles recomendadados (4,0 picoCuries) ${ }^{(8)}$.

Otros factores a tomar en cuenta en relación al cáncer de pulmón son los aspectos genéticos, terreno de cancerización familiar, la exposición a partículas de materia, la exposición a uranio, pesticidas, asbestos, 
hidrocarburos aromáticos policíclicos, arsénico y finalmente el virus papiloma humano ${ }^{(9)}$.

Existen, además, publicaciones científicas que emplean el termino "tumor de cicatriz" (Scar Cancer) para denominar aquellos tumores, resultado de la relación entre la degeneración maligna de una cicatriz pulmonar que, por lo general, son tumores periféricos con histología relacionada al adenocarcinoma.

\section{GENÉTICA Y BIOLOGÍA MOLECULAR}

El cáncer de pulmón, al igual que muchos otros tipos de cáncer, presenta múltiples cambios en la secuencia del ADN (mutaciones) y anomalías en la expresión genética (alteraciones epigenéticas), que generalmente se inician en una clona celular. Todas estas anomalías juntas resultan en la activación de oncogenes e inactivación de genes supresores de tumores y reparadores de ADN (10). La alteración genética más frecuente en cáncer de pulmón en esta vía es la mutación de EGFR, que permite a los tumores ser independientes de las señales de supervivencia transducidas por otros genes. Se mencionan mecanismos con receptores y transductores de señales bioquímicas, genes supresores de tumores (como el P53), la vía p16INK4a-Ciclina D1-CDK4$\mathrm{RB}$, la señalización del factor beta del crecimiento transformante, la deleción de $3 p$ y los genes reparadores del ADN ${ }^{(11)}$. Los marcadores moleculares que actualmente están en desarrollo son el EGFR, KRAS, ERRC1/RRM1, VEGF, Alk y MET.

\section{SIGNOS Y SÍNTOMAS}

El cáncer pulmonar temprano rara vez da síntomas, durante estos estadios el $80 \%$ de los pacientes presenta síntomas generales e inespecíficos como astenia, hiporexia y pérdida de peso. Para cuando el paciente acude a consulta se encuentra en estadios avanzados; los motivos de consulta son por lo general tos, disnea, disfonía, hemoptisis y el dolor torácico; en ocasiones, sin embargo, se pueden encontrar presentaciones específicas como el síndrome oclusivo de vena cava superior, el síndrome de Pancoast o el síndrome de Claude-Bernard-Horner ${ }^{(12)}$. Las molestias de pacientes con enfermedad metastásica están determinadas principalmente por los sitios específicos afectados tales como hueso, cerebro, hígado y glándulas adrenales ${ }^{(13)}$. Los síndromes paraneoplásicos son el conjunto de signos o síntomas no asociados a efectos mecánicos del tumor o sus metástasis y que se deben a la producción de mediadores bioquímicos ${ }^{(14)}$.
Tabla 1. Clasificación del adenocarcinoma de pulmón (IASLC/ATS/ERS).

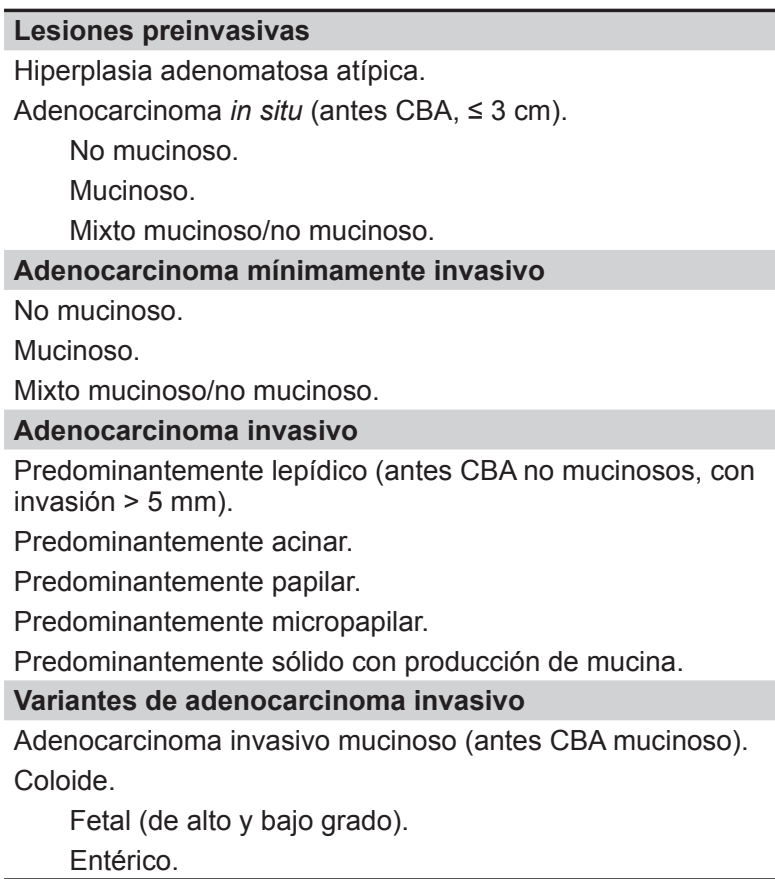

IASLC: International Association for the Study for Lung Cancer; ATS: American Thoracic Society; ERS, European Respiratory Society CBA: carcinoma bronquiolo alveolar.

\section{HISTOLOGÍA}

En el 2004, la Organización Mundial de la Salud y la Asociación Internacional para el Estudio del Cáncer de Pulmón adoptaron la misma clasificación histológica del cáncer de pulmón, la cual establece dos grandes grupos: cáncer de pulmón de células pequeñas y cáncer de pulmón de células no pequeñas (15). Descubrimientos recientes y grandes avances en las áreas de la oncología médica, la radiología y la biología molecular ${ }^{(16)}$, han llevado a la necesidad de realizar modificaciones en la clasificación actual de la International Association for the Study of Lung Cancer. El adenocarcinoma es el tipo histológico más común de cáncer pulmonar, este comprende a un grupo de neoplasias con una morfología muy heterogénea, en las que el receptor del factor de crecimiento epidérmico parece cumplir un rol importante, ya que está presente en un importante porcentaje de estas (Tabla 1).

\section{DETECCIÓN TEMPRANA}

Los proyectos de despistaje masivo del cáncer de pulmón que se están llevando a cabo en varias instituciones de manera individual o corporativa, permitirán realizar diagnósticos en etapas tempranas, 
con buenos resultados de sobrevida luego del tratamiento quirúrgico. La incorporación de la tomografía espiral multicorte de baja dosis como método de despistaje ha permitido diseñar ensayos clínicos que se espera tengan éxito en el curso de los siguientes años (17-19). Los avances en biología molecular, en genética, los estudios radiológicos y los nuevos exámenes de imágenes, como la broncoscopía con autofluorescencia o la navegación electromagnética, harán posible detectar displasias, cáncer in situ, tumores micronodulares pulmonares menores de $10 \mathrm{~mm}$ o menores de $2 \mathrm{~cm}$ (T1a).

La comunidad de investigadores está tratando de encontrar la herramienta más efectiva para la detección temprana, sea por imágenes o biomarcadores moleculares. Los primeros estudios realizados en los EE. UU. reclutaron a 30000 fumadores, en quienes se intentó detectar tempranamente el cáncer de pulmón con radiografías semestrales secuenciales y papanicolau de esputo durante un seguimiento de varios años, sin el éxito esperado ${ }^{(19)}$. Actualmente, los esfuerzos de la comunidad científica dedicada a este problema se encuentra concentrada en demostrar que la tomografía helicoidal de dosis bajas, los estudios moleculares y los biomarcadores séricos pueden ser empleados en la en la detección temprana del cáncer de pulmón ${ }^{(20,21)}$.

\section{DIAGNÓSTICO POR IMÁGENES}

El uso de tomografía computarizada ha mejorado la detección de micronódulos tumorales y la detección temprana, permitiendo establecer diferencias radiológicas entre los adenocarcinomas, adecarcinomas in situ, y carcinomas epidermoides ${ }^{(22)}$. La resonancia mangnética es superior a la tomografía computarizada para el diagnóstico de las lesiones adyacentes a la pared torácica y sulcus superior, y para la evaluación ganglionar mediastinal, detectable hasta en el $40 \%$ al momento del diagnóstico, dependiendo del tamaño, localización y tipo de lesión primaria.

La gammagrafía ósea es un método sensible en la detección de las metástasis óseas, presenta gran disponibilidad, explora el cuerpo entero en una sola prueba, tiene un costo razonable y es 50 a $80 \%$ más sensible que la radiografía para detectar metástasis esqueléticas. En contraste con la imagen radiográfica convencional, que proporciona una información anatómica, la gammagrafía aporta una información funcional con lo cual es capaz de detectar anormalidades que aún no han experimentado una alteración morfológica ${ }^{(23)}$.
La ecosonografía broncoscópica y transesofágica permite evaluar la presencia de ganglios mediastinales y si hay invasión extranodal en ellos; igualmente, puede identificar ganglios hiliares y nódulos pulmonares adyacentes a los bronquios.

La tomografía de emisión de positrones con fusión a la tomografía computarizada aporta un valor adicional al de la suma de ambas técnicas por separado. Esta herramienta diagnóstica detecta el aumento del metabolismo de la glucosa del tejido tumoral, relaciona con la actividad biológica y la mide en unidades SUV (standarized uptake value), permitiendo diferenciar de manera cuantitativa la posible presencia de neoplasia maligna, es útil para la evaluación del estadio clínico y la localización tumoral somática, excepto en el cerebro ${ }^{(24)}$.

\section{CONCEPTO DEL ESTADIO CLÍNICO}

El estadio clínico del cáncer de pulmón permite cuantificar la agresión de la enfermedad, el intercambio de información, la elegibilidad quirúrgica, diseñar el tratamiento, valorar resultados al término del tratamiento y el seguimiento de la enfermedad (25); se considerará a partir del diagnóstico citohistológico. La clasificación TNM de los tumores malignos describe la extensión del cáncer en el cuerpo de un paciente: $\mathrm{T}$ describe al tumor primario; $\mathrm{N}$ evalúa los regionales linfáticos, y $\mathrm{M}$ describe la metástasis ${ }^{(26)}$. Las nuevas técnicas de estudios por imágenes, aspectos moleculares, genéticos del cáncer, nuevas corrientes terapéuticas, entre otros, han sido incluidas en los criterios de la nueva clasificación (Tabla 2).

Tabla 2. Clasificación TNM del cáncer de pulmón.

\begin{tabular}{lcccc}
\hline & N0 & N1 & N2 & N3 \\
\hline T1 $(\leq 2 \mathrm{~cm})$ & IA & IIA & IIIA & IIIB \\
\hline T1 $(>2 \mathrm{~cm} \leq 3 \mathrm{~cm})$ & IA & IIA & IIIA & IIIB \\
T2 $(>3 \mathrm{~cm} \leq 5 \mathrm{~cm})$ & IB & IIA & IIIA & IIIB \\
\hline T2 $(>5 \mathrm{~cm}<7 \mathrm{~cm})$ & IIA & IIB & IIIA & IIIB \\
\hline T2 $(\geq 7 \mathrm{~cm})$ & IIB & IIIA & IIIA & IIIB \\
\hline T3 (invasión directa) & IIB & IIIA & IIIA & IIIB \\
\hline T3 (nódulos en el mismo lóbulo) & IIB & IIIA & IIIA & IIIB \\
\hline T4 (extensión) & IIIA & IIIA & IIIB & IIIB \\
\hline T4 (nódulos ipsilaterales) & IIIA & IIIA & IIIB & IIIB \\
\hline M1a (efusión pleural) & IV & IV & IV & IV \\
M1a (nódulos contralaterales) & IV & IV & IV & IV \\
M1b (metástasis a distancia) & IV & IV & IV & IV \\
\hline
\end{tabular}

Fuente: National Cancer Institute (disponible en: www.cancer.gov) 


\section{METODOLOGÍA DIAGNÓSTICA}

La broncoscopia permite un rápido diagnóstico y es muy útil cuando existen signos directos de neoplasia en el canal endobronquial; se puede obtener una biopsia dirigida transbronquial y una biopsia del tumor visible con fórceps, con lo cual se puede evaluar la extensión de la enfermedad bajo visión directa. Los procedimientos terapéuticos inluyen braquiterapia, radiofrecuencia, argón plasma, crioterapia y yag ( $\mathrm{Nd}$ laser, stents traqueobronquiales) (27); además de la ultrasonografía endoscópica bronquial (EBUS) o ecobroncoscopía, que es necesaria para evaluar el tumor primario, la vecindad y los ganglios regionales (grupos 2, 3, 4, 5, 7, 10 y 11). Por otra parte, el minitransductor del EBUS tiene capacidad ejecutiva para realizar el estudio bajo el sistema radial, $360^{\circ}$ y la evaluación sectorial (28), asi mismo, la ultrasonografía esofágica mediante esofagoscopía se complementa a la ecobroncoscopia. Con la navegación electromagnética, con el apoyo de la tomografía computarizada de buena calidad con un adecuado software, se consigue realizar una excelente identificación de la patología traqueobronquial y nódulos periféricos ${ }^{(29)}$. La broncoscopía de autofluorescencia, con instrumentos modernos añaden un análisis espectral de la luz dispersada verde (light scattering) ${ }^{(30)}$. La mediastinoscopía cervical anterior explora los grupos mediastinales 4R, 4L y grupo 7. Ginsberg ${ }^{(31)}$ mejoró la técnica al introducir la mediastinoscopía extendida, para abordar los grupos 5 y 6 . Posteriormente, se ha incorporado la linfadenectomía mediastinal asistida por video y linfadenectomía mediastinal transcervical extendida ${ }^{(32,33)}$. La videotoracoscopía asistida con incisiones mínimas está indicada en efusión pleural, la infiltración pleural, el compromiso pulmonar tumoral, el ganglio mediastinal y la efusión pericárdica ${ }^{(34)}$.

La biopsia por aspiración con aguja fina, ampliamente utilizada en cáncer de pulmón, es un método de diagnóstico sencillo, rápido y con mínimas complicaciones en manos expertas (35); tiene alta sensibilidad, especificidad y diagnóstica, las complicaciones más frecuentes son el neumotórax, el dolor torácico y la hemoptisis, estas se presentan solo en el $5 \%$ de los casos ${ }^{(36)}$.

\section{TRATAMIENTO}

\section{EVALUACIÓN PREQUIRÚRGICA}

La espirometría es un examen de rutina para cirugía del pulmón. Las curvas de flujo volumen permiten controlar el esfuerzo de la espiración máxima

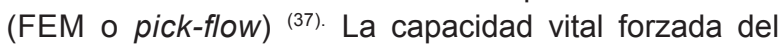

primer segundo (VEF1), mínima para poder realizar neumonectomía, deberá ser mayor de 2,0 L, para la lobectomía debe ser más de $1,5 \mathrm{~L}$, y el valor predictivo posquirúrgico de más de $0,8 \mathrm{~L}$. La capacidad de difusión pulmonar del CO con respiración, cuya sigla es DLCOsb (single-breath CO diffusing capacity of the lung), mide cuán efectivamente los gases, como el oxígeno, cumplen con la difusión alveolo capilar. En casos límites permite decidir la cirugía dependiendo de los resultados del estudio. Actualmente, las guias clínicas recomiendan la inclusión de este examen para la valoración funcional pulmonar ${ }^{(38)}$. La gammagrafía pulmonar de ventilación perfusión evalúa la reserva funcional, el porcentaje de tejido pulmonar comprometido y el no funcionante; el riesgo cardiológico, trascendental para evaluar arritmias cardiacas, valvulopatías, bloqueos, la hipertensión arterial, etc., y que podría ser motivo de contraindicaciones relativas o absolutas para la cirugía. El score de evaluación utilizado y recomendado para estos casos va a depender de la escala y puntuación del riesgo para valorar el riesgo cardiaco ${ }^{(39)}$.

\section{CIRUGÍA}

El cáncer pulmonar de células no pequeñas (CPCNP) con enfermedad temprana, T1aNOM0, tiene buena sobrevida y alta tasa de curación con cirugía. Solo los estadios clínicos I y II son elegibles para cirugía ${ }^{(40)}$; los estadios IIIA deben recibir tratamiento multidisciplinario con participación del oncólogo clínico para la quimioterapia; el radiooncólogo, para la radioterapia local y regional del tumor en forma concurrente (sincrónica) o telecrónica (consecutiva); seguida de cirugía en aquellos que demuestren buena respuesta terapéutica de la quimioradiación. Los pacientes en estadio IIIB, por lo general, recibirán tratamiento paliativo $y$, en casos excepcionales, se considerará la cirugía. Finalmente, aquellos en estadio clínico IV deberán recibir tratamiento paliativo sea con quimioterapia, radioterapia o combinación de ambos.

Solo en aquellos pacientes que presenten un nódulo pulmonar de pequeñas dimensiones y con metástasis cerebral única, periférica de pequeñas dimensiones, se contemplará la posibilidad de cirugía tanto del tumor primario como de la metástasis cerebral, seguida de radioterapia holocraneal más quimioterapia sistémica y sin tumor visible mediante los exámenes por imágenes. El tratamiento paliativo con quimioterapia, radioterapia - combinación de ambos para EC IV, incluye, tratamiento biológico, paliación con láser, argón plasma, radiofrecuencia, colocación de stent endobronquial, o manejo paliativo y manejo del dolor simplemente.

El cáncer de pulmón de células pequeñas (CPCP) puede tener indicación quirúrgica, generalmente frente 
a micromódulos o nódulos solitarios sin evidencia de metástasis ganglionar intrapulmonar, hiliar o mediastinal ${ }^{(41)}$. Se ha considerado la administración de quimioterapia convencional al encontrarse metástasis ganglionar regional en el estudio patológico ${ }^{(42)}$. Se comparó las combinaciones de dosis intensivas de cisplatino, vincristina, doxorubicina, vincristina/etoposido y cisplatino (CAV/EP). La radiación complementaria al primario y ganglios regionales está considerada así como la radioterapia cerebral profiláctica.

\section{QUIMIOTERAPIA}

La quimioterapia en el CPCNP tiene indicación en los estadíos clínicos III y IV. Los primeros estudios randomizados para la quimioterapia neoadyuvante como ensayos clínicos fase III, datan de 1994, los cuales fueron positivos a favor de la quimioterapia neoadyuvante; sin embargo, el número reducido de pacientes (60 pacientes en cada estudio) hicieron que sus resultados sean cuestionables ${ }^{(43,44)}$. Un último metaanálisis que evaluó la quimiorradioterapia concurrente en contraste con la secuencial evaluó seis estudios randomizados que incluyó a 1205 pacientes con CPCNP localmente avanzado, se demostró que la terapia concurrente tuvo una reducción relativa de la mortalidad ${ }^{(45)}$. Diversos estudios han probado que la combinación con dos drogas es superior a las terapias con una y tres drogas ${ }^{(46-51)}$. Los agentes recomendados en segunda línea son docetaxel, erlotinib, gefitinib o pemetrexed.

\section{RADIOTERAPIA}

La radioterapia puede estar indicada como tratamiento neoadyuvante o adyuvante; como tratamiento primario en enfermedad no resecable o médicamente inoperable; y como terapia paliativa. En el CPCNP solo la radioterapia conformacional 3D (RTC3D) con trazado del volumen blanco con tomografía computarizada permite la planificación adecuada para la radioterapia de dosis alta. En tanto que la radioterapia estereotáxica se considera para paciente con lesiones inoperables en estadio clínico I con lesiones periféricas y ganglios negativos, estas lesiones deben ser menores a $5 \mathrm{~cm}$ en su máxima extensión ${ }^{(52,53)}$. La irradiación profiláctica del sistema nervioso central mejora las tasa de supervivencia global y libre de enfermedad en pacientes que han alcanzado una remisión completa, igualmente, reduce el riesgo de metástasis cerebrales ${ }^{(54)}$. En las metástasis cerebrales se indica dosis altas y fraccionadas ${ }^{(5)}$. En pacientes con metástasis única con tumor primario controlado se prefieren esquemas de hiper fraccionamiento combinados con cirugía ${ }^{(56)}$, en tanto que la compresión medular requiere intervención multidisciplinaria además de la radioterapia ${ }^{(57)}$. La radiación en las metástasis óseas con esquemas de fraccionamiento consigue buenos resultados.

\section{MICROARRAYS}

Gracias a su rol en la generación de información sobre la secuenciación sistemática de genomas completos el uso de los microarrays en oncología es cada vez más importante. En cáncer de pulmón es útil para detección y diagnóstico de genes que se activan o se reprimen en distintas condiciones, asi como para la detección de las amplificaciones y deleciones de ADN que contribuyen al desarrollo y progresión en este tipo de cáncer. En el CPCNP, gracias a esta tecnología, se han identificado como importantes en el desarrollo y progresión a los genes EGFR, HER2, p53, RRM1 y ERCC1; el análisis de estos genes en los pacientes con este cáncer puede predecir su supervivencia, y su utilidad está considerada en la respuesta al tratamiento. En el CPCP ayuda a identificar alteraciones novedosas y es capaz de establecer hibridación genómica comparativa ${ }^{(58)}$.

Con esta plataforma tecnológica se vienen desarrollando beneficios sustanciales en el campo genético, expresión de genes antiapoptóticos, represión transcripcional, carcinogenicidad, agresión tumoral, comportamiento de la respuesta terapéutica frente a la quimioterapia, blancos moleculares (tratamiento personalizado) y pronóstico.

\section{CONCLUSIONES}

El cáncer de pulmón es una neoplasia prevalente entre los tumores malignos, con mayor frecuencia de presentación en el sexo masculino, sin embargo, en aumento en el sexo femenino. La mayor vinculación carcinogenética está en relación al tabaco, otras causas son la exposición a humo de leña doméstico, asbestos, hidrocarburos, radiaciones, entre otros. Los estudios genéticos establecen que las células malignas del cáncer de pulmón presentan mutaciones y deleciones en genes determinados, las que son cada vez mejores entendidas. El tipo histológico más frecuente es el adenocarcinoma, cuya clasificación histológica ha sido modificada recientemente. La detección temprana de micronódulos, con el uso de tomografía espiral multicorte de bajas dosis, se viene evaluando en poblaciones de alto riesgo, mediante estudios prospectivos, cuyos resultados aún no han concluido. El diagnóstico con la ayuda de imágenes es fundamental y los estudios de extensión de enfermedad mejoran con los avances de la imagenología, incluyendo técnicas de tomografía, medicina nuclear y tomografía con emisión de proteínas con fusión a la tomografía computarizada que permite la 
clasificación TNM por estadios clínicos. El diagnóstico citohistológico es fundamental para precisar la naturaleza y pronóstico de la enfermedad. El tratamiento quirúrgico en estadios tempranos tiene una excelente sobrevida en pacientes considerados elegibles luego de la evaluación del riesgo quirúrgico, de reserva pulmonar, entre otros. El tratamiento multidisciplinario que incluye la cirugía, la quimioterapia y la radioterapia se ha considerado en estadios moderadamente avanzados. La quimioterapia y radioterapia tienen un rol de paliación en estadios avanzados, con criterio de calidad de vida y mejora de la sobrevida, eventualmente.

Fuentes de financiamiento: autofinanciado.

Conflictos de interés: el autor declara no tener conflictos de interés en la publicación de este artículo.

\section{REFERENCIAS BIBLIOGRÁFICAS}

1. National Comprehensive Cancer Network (NCCN). Non-small cell lung cancer [Internet]. Fort Washington: NCCN; 2011 [citado el 12 de octubre de 2011]. Disponible en: http://www.nccn.org/professionals/physician_gls/f_guidelines.asp

2. International Agency for Research on Cancer. GLOBOCAN 2008 [Internet]. Lyon: OMS; c2010 [citado el 13 de noviembre de 2012]. Disponible en: http://globocan.iarc.fr/factsheet. asp\#BOTH

3. Wilkins N, Yürekli A, Hu T. Análisis económico de la demanda de tabaco. Washington, DC: OPS/Banco Mundial; 2004.

4. Organización Mundial de la Salud. Control del tabaco: reforzar las actividades nacionales. En: Informe sobre la salud en el mundo 2003. Ginebra: OMS; 2003. p. 100-4.

5. Barrueco Ferrero M, Hernández Mezquita MA, Torrecilla García M. Manual de prevención y tratamiento del tabaquismo. Madrid: ERGON; 2003.

6. Bach PB, Jett JR, Pastorino U, Tockman MS, Swensen SJ, Begg CB. Computed tomography screening and lung cancer outcomes. JAMA. 2007;297(9):953-61.

7. Desai MA, Metha $S$, Smith KR. Indoor smoke from solid fuels. Assessing the environmental burden of disease at national and local levels. Geneva: World Health Organization; 2004.

8. Delgado J, Martinez LM, Sánchez TT, Ramirez A, Iturria C, González-Avila G. Lung cancer pathogenesis associated with wood smoke exposure. Chest. 2005;128(1):124-31.

9. Gorlova OY, WengSF, Zhang Y, Amos CI, Spitz MR. Aggregation of cancer among relatives of never-smoking lung cancer patients. Int J Cancer. 2007;121(1):111-8.

10. Coyle YM, Minahjuddin AT, Hynan LS, Minna JD. An ecological study of the association of metal air pollutants with lung cancer incidence in Texas. J Thorac Oncol. 2006;1(7):654-61.
11. Field JK, Youngson JH. The Liverpool Lung Proyect: molecular epidemiological study of early lung cancer detectión. Eur Respir J. 2002;20(2):464-79.

12. Zhang Z, Stiegler AL, Boggon TJ, Kobayashi S, Halmos B. EGFR-mutated lung cancer: a paradigm of molecular oncology. Oncotarget. 2010;1(7):497-514.

13. Fossella FV, Komaki R, Putnam JB Jr, Lung cancer. New York: Springer; 2002.

14. Hansen H. Textbook of Lung Cancer. 2nd Edition. London Informa Healthcare. 2008.

15. Defranchi SA, Cassivi SD, Nichols FC, Allen MS, Shen KR. N2 disease in T1 non-small cell lung cancer. Ann Thorac Surg. 2009;88(3):924-8.

16. Boyle P, Levin B. World Cancer Report 2008. Lyon: International Agency for Research on Cancer; 2008.

17. National Lung Screening Trial Research Team, Aberle DR, Adams AM, Berg CD, Black WC, ClappJD, et al. Reduced lungcancer mortality with low-dose computed tomographic screening. N Engl J Med. 2011;365(5):395-409.

18. Greaves SM, Brown K, Garon EB, Garon $\mathrm{BL}$. The new staging system for lung cancer: imaging and clinical implications. J Thorac Imaging. 2011;26(2):119-31.

19. Camilo R, Capelozzi VL, Siqueira SA, Del Carlo Bernardi F. Expression of p63, keratin $5 / 6$, keratin 7 , and surfactant-A in non-small cell lung carcinomas. Hum Pathol. 2006;37(5):542-6.

20. International Early Lung Cancer Action Program Investigators, Henschke CI, Yankelevitz DF, Libby DM, Pasmantier MW, Smith JP, et al. Survival of patients with stage I lung cancer detected on CT screening. N Engl J Med. 2006;355(17):1763-71.

21. Sone S, Takashima S, Li F, Yang Z, Honda T, Maruyama Y, et al. Mass screening for lung cancer with mobile spiral computed tomography scanner. Lancet. 1998;351(9111):1242-5.
22. Gill RR, Matsusoka S, Hatabu H. Cavities in the lung in oncology patients: imaging overview and differential diagnoses. Applied Radiology. 2010;39(6):10-21.

23. Baum RP, Hellwig D, Mezzetti M. Position of nuclear medicine modalities in the diagnostic workup of cancer patients: lung cancer. QJ Nucl Med Mol Imaging. 2004;48(2):119-42.

24. Lardinois D, Weder W, Hany TF, Kamel EM, Korom S, Seifert B, et al. Staging of non-small-cell lung cancer with integrated positron-emission tomography and computed tomography. N Engl J Med. 2003;348(25):2500-7

25. Sobin LH, Gospodarowicz MK, Wittekind Ch (eds). TNM classification of malignant tumors. 7th ed. Oxford: Wiley-Blackwell; 2009.

26. Mountain CF. Revisions in the international system for staging lung cancer. Chest. 1997;111(6):1710-7.

27. British Thoracic Society Bronchoscopy Guidelines Committee, a Subcommittee of Standards of Care Committee of British Thoracic Society. British Thoracic Society guidelines on diagnostic flexible bronchoscopy. Thorax. 2001;56 Suppl 1:i1-21.

28. Herth F, Krasnik M, Yasufuku K, Rintoul R, Ernst A. Endobronchial ultrasoundguided transbronchial needle aspiration. J Bronchol. 2006;13(2):84-91.

29. Gildea TR, Mazzone PJ, Karnak D, Meziane M, Mehta AC. Electromagnetic navigation diagnostic bronchoscopy: a prospective study. Am J Respir Crit Care Med. 2006;174(9):982-9.

30. Ikeda N, Honda H, Katsumi T, Okunaka T, Furukawa K, Tsuchida T, et al. Early detection of bronchial lesions using lung imaging fluorescence endoscope. Diagn Ther Endosc. 1999;5(2):85-90.

31. Ginsberg RJ, Rice TW, Goldberg H, Waters PF, Schmoker BJ.Extended cervical mediastinoscopy: a single staging procedure for bronchogenic carcinoma of the left upper lobe. J Thorac Carciovasc Surg. 1987;94(5):673-8. 
32. Kuzdzał J, Zieliński M, Papla B, Szlubowski A, Hauer $€$, Nabiałek T, et al. Transcervical extended mediastinal lymphadenectomy--the new operative technique and early results in lung cancer staging. Eur J Cardiothorac Surg. 2005;27(3):384-90.

33. Leschber G, Holinka G, Linder A. Video-assisted mediastinoscopic lymphadenectomy (VAMLA)--a method for systematic mediastinal lymphnode dissection. Eur J Cardiothorac Surg. 2003;24(2):192-5.

34. Rubini C. Biopsia intratorácica percutánea por aspiración. Experiencia en el Instituto Nacional de Enfermedades Neoplásicas. Acta Med Per. 1995;13(3).

35. Pantzar P, Meurala H, Koivuniemi A, Laustela E. Peroperatorive fine needle aspiration biopsy of lung tumors. Scand J Thorac Cardiovas Surg. 1983;17(1):51-3.

36. He J, Shao W, Cao C, Yan T, Wang D, Xiong XG, et al. Long-term outcome and cost-effectiveness of complete versus assisted video-assisted thoracic surgery for non-small cell lung cancer. J Surg Oncol. 2011;104(2):162-8

37. Ferguson GT, Enright PL, Buist AS, Higgins MW. Office spirometry for lung health assessment in adults: A consensus statement from the National Lung Health Education Program. Chest. 2000;117(4):1146-61.

38. Brunelli A, Charloux A, Bolliger CT, Rocco G, Sculier JP, Varela G, et al.ERS/ESTS clinical guidelines on fitness for radical therapy in lung cancer patients (surgery and chemo-radiotherapy). Eur Respir J. 2009;34(1):17-41.

39. Lassen U, Osterlind $K$, Hansen $M$, Dombernowsky P, Bergman B, Hansen $\mathrm{HH}$. Long-term survival in small-cell lung cancer: posttreatment characteristics in patients surviving 5 to $18+$ years--an analysis of 1,714 consecutive patients. J Clin Oncol. 1995 May;13(5):1215-20.

40. Savino J, Del Lous A. Valoración preoperatoria del individuo con alto riesgo quirúrgico. Clin Quir Norte Am. 1985;4:779-809.

41. Prasad US, Naylor AR, Walker WS, Lamb D, Cameron EW, Walbaum PR. Long-term survival after pulmonary resection for small cell carcinoma of the lung. Thorax. 1989;44(10):784-7.

42. Landreneau RJ, Sugarbaker DJ, Mack MJ, Hazelrigg SR, Luketich JD, Fetterman L, et al. Wedge resection versus lobectomy for stage I (T1 N0 M0) non-smallcell lung cancer. J Thorac Cardiovasc Surg. 1997;113(4):691-700.

43. Rosell R, Gómez-Codina J, Camps C, Javier Sánchez J, Maestre J, Padilla J, et al. Preresectional chemotherapy in stage IIIA nonsmall cell lung cancer: a 7-years assessment of a randomized controlled trial. Lung Cancer. 1999;26(1):7-14.

44. Roth JA, Fossella F, Komaki R, Ryan MB, Putnam JB Jr, Lee JS, et al. A randomised trial comparing perioperative chemotherapy and surgery with surgery alone in resectable stage IIIA non-small cell lung cancer. J Natl Cancer Inst. 1994;86(9):673-80.

45. MacManus MP, Hicks RJ, Matthews JP, Hogg A, McKenzie AF, Wirth A, et al. High rate of detection of unsuspected distant metastases by PET in apparent stage III non-small cell lung cancer: implications for radical radiation therapy. Int J Radiat Oncol Biol Phys. 2001;50(2):287-93.

46. Goldstraw P, Crowley J, Chansky K, Giroux DJ, Groome PA, Rami-Porta R, et al. The IASLC Lung Cancer Staging Project: proposals for the revision of the TNM stage groupings in the forthcoming (seventh) edition of the TNM Classification of malignant tumours. J Thorac Oncol. 2007;2(8):706-14.

47. NSCLC Meta-Analyses Collaborative Group. Chemotherapy in addition to supportive care improves survival in advanced non-small-cell lung cancer: a systematic review and meta-analysis of individual patient data from 16 randomized controlled trials. J Clin Oncol. 2008;26(28):4617-25.

48. Schiller JH, Harrington D, Belani CP, Langer C, Sandler A, Krook J, et al. Comparison of four chemotherapy regimens for advanced non-small-cell lung cancer. N Engl J Med. 2002;346(2):92-8.

49. Georgoulias V, Ardavanis A, Agelidou A, Agelidou M, Chandrinos V, Tsaroucha $\mathrm{E}$, et al. Docetaxel versus docetaxel plus cisplatin as front-line treatment of patients with advanced non-small-cell lung cancer: a randomized, multicenter phase III trial.J Clin Oncol. 2004;22(13):2602-9.

50. Davies AM, Lara PN, Lau DH. Intermittent erlotinib in combination with docetaxel (DOC): phase I schedules designed to achieve pharmacodynamic separation. Program and abstracts of the 41st Annual
Meeting of the American Society of Clinical Oncology. 2005. p. 13-7.

51. Aoyama H, Shirato H, Tago M, Nakagawa K, Toyoda T, Hatano K, et al. Stereotactic radiosurgery plus wholebrain radiation therapy vs stereotactic radiosurgery alone for treatment of brain metastases: a randomized controlled trial. JAMA. 2006;295(21):2483-91.

52. Onishi H, Araki T, Shirato H, Nagata Y, Hiraoka M, Gomi K, et al. Stereotactic hypofractionated high-dose irradiation for stage I nonsmall cell lung carcinoma: clinical outcomes in 245 subjects in a Japanese multiinstitutional study. Cancer. 2004;101(7):1623-31.

53. Bradley J, Graham MV, Winter K, Purdy JA, Komaki R, Roa WH, et al. Toxicity and outcome results of RTOG 9311: a phase I-II dose-escalation study using three-dimensional conformal radiotherapy in patients with inoperable non-small-cell lung carcinoma. Int J Radiat Oncol Biol Phys. 2005;61(2):318-28.

54. De las Heras García B, Jiménez Gordo AM, Casado Saenz E, Zamora Auñón P Cantalejo Moreira M, González Barón M. Síndrome de vena cava superior: presentación de seis casos. An Med Interna (Madrid). 2001;18:369-72.

55. Al-Shamy G, Sawaya R. Management of brain metastases: the indispensable role of surgery.J Neurooncol. 2009;92(3):275-82.

56. Timmerman R, McGarry R, Yiannoutsos C, Papiez L, Tudor K, DeLuca J, et al. Excessive toxicity when treating central tumors in a phase II study of stereotactic body radiation therapy for medically inoperable early-stage lung cancer. J Clin Oncol. 2006;24(30):4833-9.

57. Miller LD, Long PM, WongL, Mukherjee S, McShane LM, Liu ET. Optimal gene expression analysis by microarrays. Cancer Cell. 2002;2(5):353-61.

58. Sugita M, Geraci M, Gao B, Powell R, Hirsch F, Johnson G, et al. Combined use of oligonucleotide and tissue microarrays identifies cancer/testis antigens as biomarkers in lung carcinoma. Cancer Res. 2002;62(14):3971-9.

Correspondencia: Edgar Amorin Kajatt Dirección: Av. Javier Prado Este 1010-306. Lima 27, Perú.

Telefono (511) 2242224 anexo 1235

Correo electrónico:edgar.amorin@gmail.com 